\title{
Long-term outcome following cranial biceps brachii tendon transposition in a dog with a traumatic cranial scapulohumeral luxation
}

\author{
Lauren N. Barber ${ }^{1}$, Daniel D. Lewis², Erin G. Porter ${ }^{2}$ and Lindsay H. Elam ${ }^{3}$ \\ ${ }^{1}$ San Diego Humane Society, San Diego, California, USA \\ ${ }^{2}$ Department of Small Animal Clinical Sciences, College of Veterinary Medicine, University of Florida, Gainesville, FL, USA \\ ${ }^{3}$ Department of Clinical Sciences, College of Veterinary Medicine and Biomedical Sciences, Colorado State \\ University, Fort Collins, Colorado, USA
}

\begin{abstract}
Background: Cranial luxation of the scapulohumeral has been rarely reported in dogs and there is limited information available regarding surgical management of this condition, particularly with respect to long-term functional outcomes. Case Description: This report describes the successful resolution of a chronic traumatic cranial scapulohumeral joint luxation in a dog that was stabilized by cranial transposition of the biceps brachii tendon of origin. At surgery, an osteotomy of the greater tubercle was performed and a trough was made in the exposed bed of the osteotomy. The transverse humeral ligament was incised, and the bicipital tendon was levered into the trough and secured in that location by reattachment of the greater tubercle using multiple Kirschner wires and a figure-of-eight tension band wire. Postoperatively, the dog was maintained in a Spica splint for 2 weeks. Although surgical reduction was performed 4 months after the original injury, the luxation did not recur and the dog did not have appreciable lameness 14 months following the surgery.
\end{abstract}

Conclusion: Although cranial transposition of the bicipital tendon is an invasive procedure, this dog's scapulohumeral luxation did not recur and the procedure yielded an excellent long-term functional outcome.

Keywords: Cranial, Dog, Luxation, Scapulohumeral joint, Surgical reduction.

\section{Introduction}

Cranial scapulohumeral luxation is an uncommon traumatic injury in dogs (Vaughn, 1967; DeAngelis and Schwartz, 1970; Vasseur et al., 1982, 1983; Vasseur and Gregory, 1985; Brinker et al., 1997). Dogs generally sustain lateral or medial scapulohumeral luxations, while cranial scapulohumeral luxations have only been sporadically reported (Vaughn, 1967; Campbell, 1968; DeAngelis and Schwartz, 1970; Vasseur et al., 1982, 1983). Although surgical techniques to address cranial scapulohumeral luxation are described in a number of texts (Campbell, 1968; DeAngelis and Schwartz, 1970; DeAngelis, 1975; Vasseur et al., 1982; Hohn et al., 1983; Vasseur et al., 1983; Newton and Nunamaker, 1985; Vasseur and Gregory, 1985; Brinker et al., 1997), there is negligible specific information detailing the clinical outcome of this procedure in affected dogs (DeAngelis and Schwartz, 1970). The objective of this report was to describe the presenting clinical abnormalities in a dog with a recurrent cranial scapulohumeral luxation and to report the long-term functional outcome (Cook et al., 2010) following open reduction and surgical stabilization of the scapulohumeral joint using cranial translocation of the tendon of origin of the biceps brachii muscle (DeAngelis, 1975; Hohn et al., 1983).

\section{Case Details}

A 1-year-old, $22 \mathrm{~kg}$, male castrated Catahoula Leopard dog was referred to the University of Florida Small Animal Hospital (UF SAH) for evaluation of an intermittent left thoracic limb lameness. Four months prior to referral, the dog was hit by a car and sustained a left scapulohumeral luxation. Digital
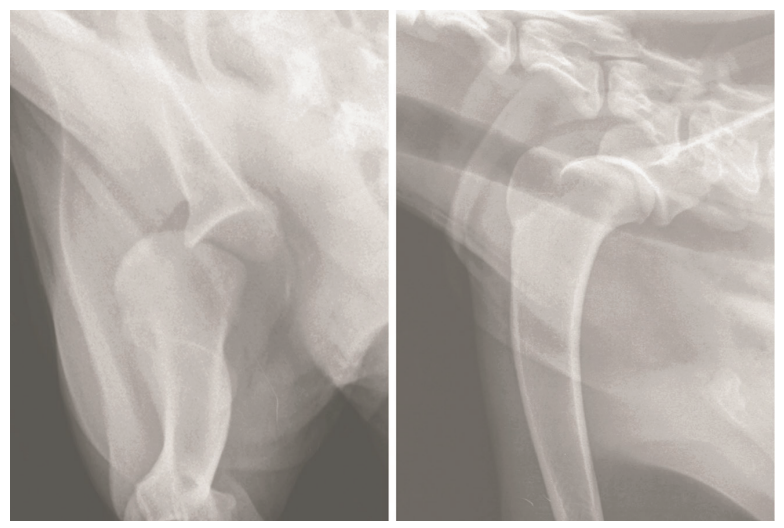

Fig. 1. Caudocranial (left) and mediolateral (right) projection radiographs of the proximal left thoracic limb obtained on the day the dog was hit by a car. The humerus is luxated laterally with slight cranioproximal displacement. 
radiographs (AGFA CR30-x generator, with $\mathrm{CR}$ MD4.0T plates) of the left thoracic limb were obtained by the referring veterinarian on the day of the initial injury. The left humeral head was laterally and slightly cranioproximally luxated from the glenoid fossa of the scapula (Fig. 1).

The luxation was reduced and the left thoracic limb was immobilized in flexion using a Velpeau sling. Carprofen (Rimadyl, Zoetis) was administered at a dose of 2.2 $\mathrm{mg} / \mathrm{kg}$ PO every 12 hours for 14 days and the owner was advised to markedly restrict the dog's activity for 3 weeks. Unfortunately, the humeral head could be palpated cranially to the scapula the following day. The luxation was again reduced and a Velpeau sling was reapplied, but the luxation recurred. This sequence of events was repeated five times over the subsequent 7 days because of recurrence of the luxation. A Spica splint was then applied following closed reduction and the splint was maintained for 1 week. The luxation recurred following splint removal and no further coaptation was applied. The scapulohumeral joint would intermittently luxate and the dog would not bear weight on the limb when the luxation occurred. The owners could readily reduce the luxation and the dog would resume weight-bearing on the limb. Two months after the initial injury, the owner surrendered the dog because of financial concerns. The dog was adopted and was primarily confined in a crate; however, the dog's left scapulohumeral joint would luxate multiple times each day. The dog would not bear weight on the limb when the luxation recurred, holding the manus proximal to the shoulder with the elbow and carpus flexed. The owner was able to manually reduce the luxation if the luxation did not spontaneously reduce. The dog seemed comfortable and ambulated normally on the limb when the joint was reduced.

At the time of presentation to the UF SAH, the dog ambulated without apparent lameness. During the orthopedic examination, flexion of the dog's left scapulohumeral joint resulted in cranial displacement of the humeral head causing the dog to maintain the limb in a flexed position. The luxation was readily reduced by extending the scapulohumeral joint and applying traction to the limb. The dog appeared agitated when the joint was luxated, but resumed normal behavior and use of the left thoracic limb when the humeral head was manually reduced.

The dog was sedated with dexdomitor $300 \mathrm{mcg}$ IV and butorphanol $0.1 \mathrm{mg} / \mathrm{kg} \mathrm{IV}$, and radiographs of the left scapulohumeral joint were obtained (Canon Imaging System, CXDI-50G digital radiography detectors, UF $\mathrm{SAH})$. The left scapulohumeral joint was congruent and abnormalities were limited to mild, smoothly margined osseous proliferation present on the lateral margin of the glenoid cavity, which was thought to represent an enthesophyte formation at the origin of the lateral glenohumeral ligament. Radiographs were not obtained when the luxation was present.
Open surgical reduction and stabilization (Vaughn, 1967; DeAngelis and Schwartz, 1970) was recommended. The dog was premedicated using dexdomitor 300 mcg IV and butorphanol $0.1 \mathrm{mg} / \mathrm{kg} \mathrm{IV}$, perioperative cefazolin was given at $30 \mathrm{mg} / \mathrm{kg}$ IV and continued Q 8 hours for 24 hours. Anesthesia was induced with 7.6 $\mathrm{mg} / \mathrm{kg}$ Propofol given over $60-90$ seconds to allow intubation, where anesthesia was maintained with Isoflurane. The left thoracic limb was clipped and prepared for aseptic surgery. The dog was positioned in dorsal recumbency and a craniolateral approach to the left scapulohumeral joint was done. During the initial stages of the procedure, the humeral head intermittently luxated cranially, predominately when the scapulohumeral joint was flexed. The acromial head of the deltoid and the sternocephalicus muscles were retracted and the infraspinatus, superficial pectoral, and deep pectoral muscles were incised and reflected to expose the joint capsule. The cranial joint capsule was circumferentially incised to expose the tendon of origin of the biceps brachii muscle and the transverse humeral ligament was incised. An osteotomy of the greater tubercle of the humerus was performed with an oscillating saw, allowing proximal reflection of the supraspinatus muscle. A pneumatic drill and burr were used to create a trapezoidal proximal-to-distal trough centrally in the exposed cancellous bone in the base of the osteotomy site. The trough was sculpted to conform to the dimensions of the bicipital tendon including the proximal myotendinous portion of the biceps brachii muscle (Fig. 2). The biceps brachii tendon was levered laterally into the trough using a Freer periosteal elevator and the humerus no longer luxated when the bicipital tendon was positioned in the trough (Fig. 3). The joint capsule was reopposed using 2-0 PDS placed in an interrupted cruciate pattern which prevented displacement of the tendon. To augment the

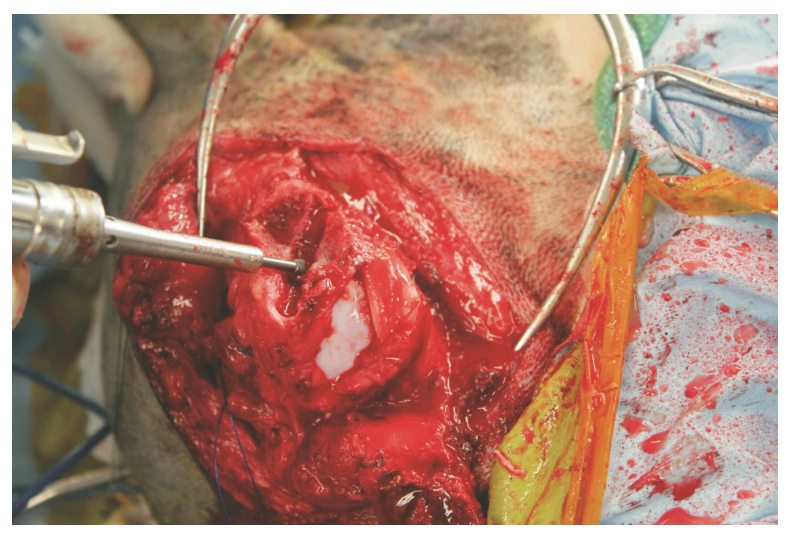

Fig. 2. Intraoperative photograph showing use of a pneumatic drill and burr to create a trough in the base of the greater tubercle osteotomy. The transverse humeral ligament is incised and the bicipital tendon is displaced medial to the intertubercular groove. 
stability afforded by the capsulorrhaphy, a six-hole $2.0 \mathrm{~mm}$ locking compression plate (DePuy Synthes, West Chester, PA) was placed along the craniolateral margin of the distal scapulohumeral joint line. The plate bridged the bicipital tendon and was secured to the humerus with three locking and one cortical 2.0 $\mathrm{mm}$ screws (Fig. 4). The greater tubercle was then repositioned on the incised surface of the humerus, $1 \mathrm{~cm}$ distal to the tubercle's original location. The tubercle was secured with two 1.6-mm Kirschner wires and two strands of 18-gauge orthopedic wires placed in a figure-of-eight configuration as a tension band. Five additional 1.6-mm Kirschner wires were placed to further secure the tuberosity to the proximal humerus (Fig. 5). The remainder of the wound was closed in a routine fashion. After obtaining post-operative radiographs (Fig. 6), the left thoracic limb was placed in a Spica splint (DeCamp et al., 2016). Postoperatively, the dog was administered methadone $(0.1 \mathrm{mg} / \mathrm{kg} \mathrm{IV}$

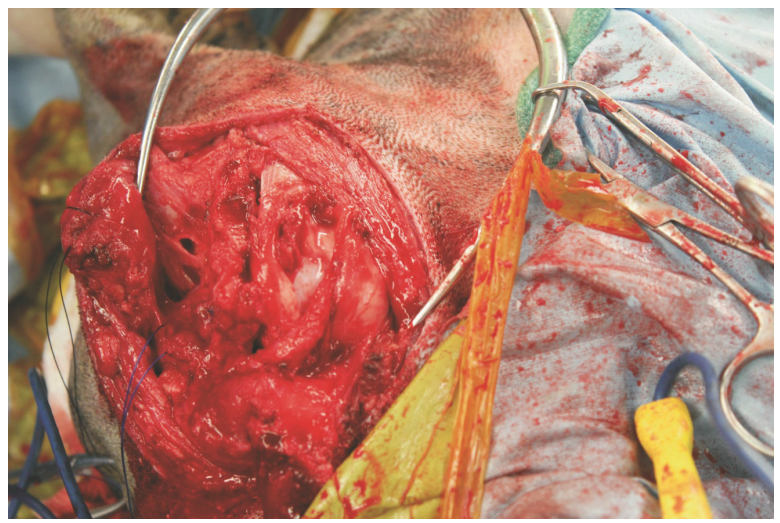

Fig. 3. Intraoperative photograph showing transposition of the bicipital tendon into the trough burred in the osteotomized surface of the greater tubercle.

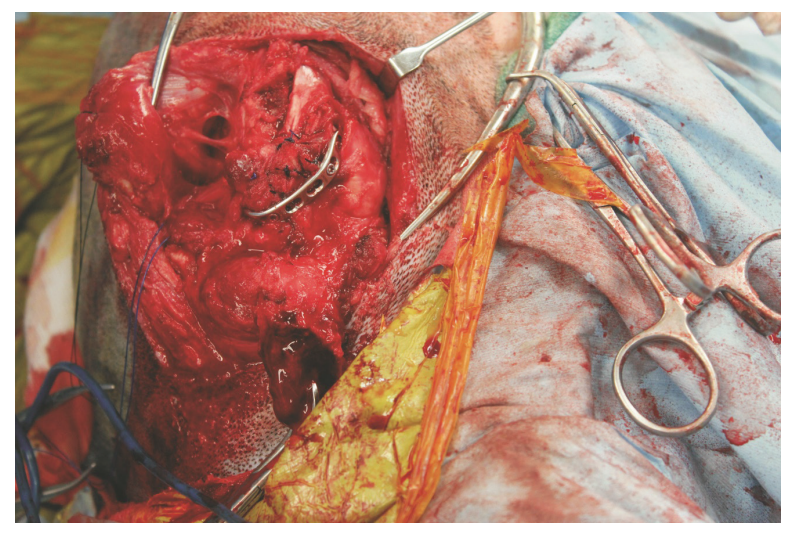

Fig. 4. Intraoperative photograph taken after closure of the joint capsule. Following capsulorrhaphy, a locking compression plate was secured to the craniolateral margin of the distal scapulohumeral joint line. Note the bicipital tendon extending distal to the sutured capsule.
Q 4 hours for two doses), transitioned to tramadol (3.3 $\mathrm{mg} / \mathrm{kg}$ PO Q 8, beginning the morning following surgery), carprofen (16 mg/kg PO Q 12, beginning in the morning following the surgery), trazodone (3.3 $\mathrm{mg} / \mathrm{kg}$ PO Q 8, beginning in the evening following the surgery), cefazolin continued ( $30 \mathrm{mg} / \mathrm{kg}$ IV Q 8 for 24 hours), transitioned to cephalexin (33 mg/kg PO Q 12, beginning in the evening following the surgery) and continued through discharge for 14 days.

The dog was discharged 2 days following the surgery and the owner was instructed to restrict the dog's activity, which consisted of confining the dog to a crate or small room in the house when unattended and taking

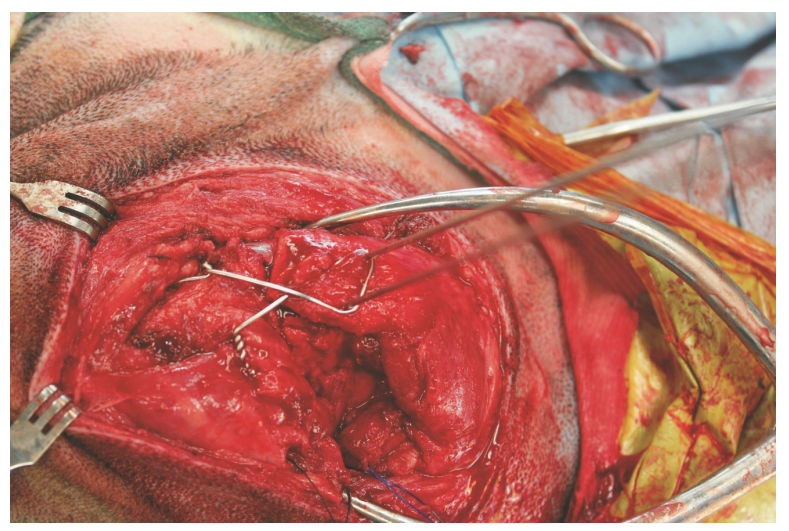

Fig. 5. Intraoperative photograph obtained after reattachment of the greater tubercle. The tubercle was translated distally and secured with Kirschner wires and a figure-of-eight tension band wire.
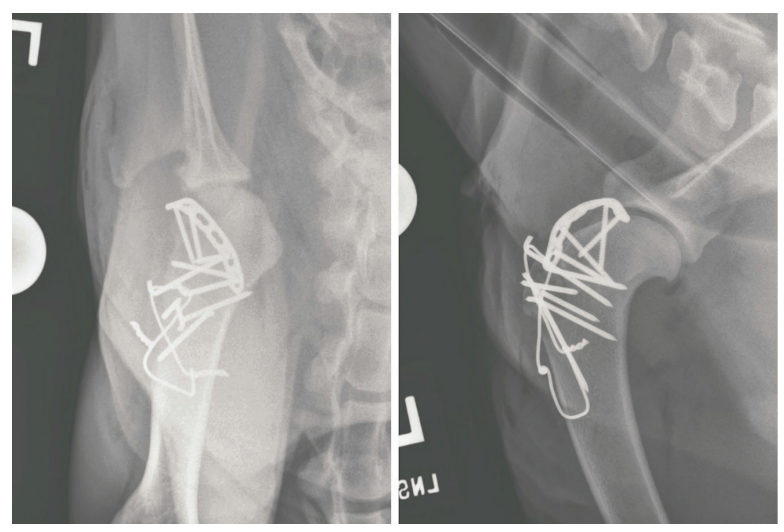

Fig. 6. Caudocranial (left) and mediolateral (right) projection radiographs of the left scapulohumeral joint obtained immediately following the surgery. The scapulohumeral joint was congruent. A contoured locking compression plate was secured to the craniolateral articular margin of the proximal humerus. The plate was placed following joint capsule closure and bridged the transposed biceps brachii tendon. The greater tubercle was attached, but transposed $1 \mathrm{~cm}$ distally, and secured using multiple Kirschner wires and a figure-ofeight tension band wire. 
the dog outside for short walks on a leash, mainly for the purposes of urination and defecation. The dog was in a moderate amount of discomfort, which improved with the administration of carprofen and tramadol, for the first 3 days at home. The dog subsequently became more comfortable, but the dog did not effectively use the limb while the Spica splint was in place.

The dog was reevaluated at the UF SAH 2 weeks following the surgery and the Spica splint was removed. The left scapulohumeral joint was stable and could not be luxated. Manipulation of the left scapulohumeral joint was mildly painful. Flexion and extension of the joint was markedly reduced. The left carpus was placed in a carpal flexion bandage (DeCamp et al., 2016) for an additional 10 days to encourage movement in the left scapulohumeral joint but prevent weight-bearing on the limb. The dog was evaluated again at the UF SAH 4 weeks following the surgery. The dog remained predominately non-weight-bearing on the left thoracic limb after removal of the carpal flexion bandage, although the dog intermittently rested the dorsum of the left manus on the ground while standing. Radiographs of the left scapulohumeral joint were obtained under sedation (dexdomitor $325 \mathrm{mcg}$ IV and butorphanol $0.1 \mathrm{mg} / \mathrm{kg}$ IV). The left scapulohumeral joint was congruent and there were no complications noted with the surgical implants. Flexion and extension of the left scapulohumeral joint was mildly improved. Extension of the left elbow was also noted to be decreased. A gradual return to normal activity was recommended and the dog was referred to the UF Integrative Medicine Service for rehabilitative therapy. A multimodal rehabilitative approach was implemented with a focus on gait retraining, strength building, and increasing shoulder and elbow joint range of motion. This was achieved through the use of underwater treadmill therapy, photobiomodulation, therapeutic ultrasound, and manual therapy. The dog was seen weekly for formal rehabilitation session with the owner performing prescribed therapeutic exercises at home between sessions. Supplementation with nutraceuticals including fish oil and a combination joint health product including glucosamine, chondroitin, methylsulfonylmethane, and avocado soybean unsaponifiables (Dasuquin, Nutramax) was initiated. By 8 weeks following the surgery, the dog had regained substantial use of the left thoracic limb and was weight-bearing during all gaits, although still moderately lame. Following each integrative therapy session, the dog was more consistently weight-bearing with a greater range of motion in the left scapulohumeral joint. The exercises and range of motion movements were continued at home for an additional month, after which time the dog resumed most normal activities including running walking 5-8 $\mathrm{km}$ per day and swimming with only nominal lameness.

The dog was reexamined at the UF SAH 14 months following the surgery to obtain long-term functional results of the procedure (Cook et al., 2010). The owner reported the dog had resumed all normal activities without lameness. On examination, the dog ambulated without appreciable lameness. The dog was walked over a force platform (Model\# OR6-6-1000; Advanced Mechanical Technology, Newton, MA) and mild asymmetry between the thoracic limbs was recorded. The peak vertical force $(100 * \mathrm{~N} / \mathrm{kg}$ body weight $)$ and vertical impulse for the left thoracic limb were $5 \%$ and $6 \%$ lesser than the right thoracic limb. The left scapulohumeral joint was comfortable on palpation and could not be luxated. The dog was sedated (dexdomitor $350 \mathrm{mcg}$ IV and butorphanol $0.1 \mathrm{mg} / \mathrm{kg} \mathrm{IV}$ ) and radiographs of the left scapulohumeral joint were obtained (Fig. 7). The left scapulohumeral joint was congruent and there was minor osteophyte formation on the caudal and lateral aspects of the glenoid cavity. Three out of the four bone screws engaging the bone plate along the proximal humerus had broken; there was moderate sclerosis of the humeral head and neck; and the humeral cortex at the level of the third most distal screw was irregularly marginated, decreased in mineral opacity, and ill-defined. The greater tubercle osteotomy had obtained union. While sedated, left forelimb circumference and joint flexion and extension angles were measured. There was mild muscle atrophy of the left thoracic limb: the circumference of the left brachium was $22.3 \mathrm{~cm}$ (right: $23.1 \mathrm{~cm}$ ) and the left antebrachium was $14.1 \mathrm{~cm}$ (right: $14.5 \mathrm{~cm}$ ). While flexion of the left scapulohumeral joint was now equal to the right (both $37^{\circ}$ ), extension of the left scapulohumeral joint was improved $\left(145^{\circ}\right)$, but still limited as compared to the right $\left(170^{\circ}\right)$. Extension $\left(152^{\circ}\right)$ and flexion $\left(18^{\circ}\right)$ of the left elbow had also improved and were comparable to the right elbow (extension $161^{\circ}$; flexion $16^{\circ}$ ).
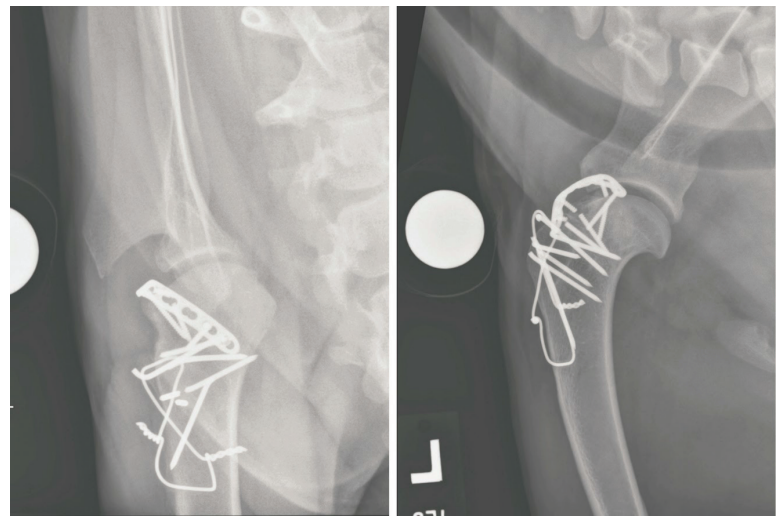

Fig. 7. Caudocranial (left) and mediolateral (right) projections of the left scapulohumeral joint made 14 months following the surgery. The scapulohumeral joint is congruent. Three bone screws engaging the contoured bone plate are fractured, and there is sclerosis of the femoral head and neck. The humeral cortex at the level of the third most distal screw is decreased in mineral opacity and ill-defined. 


\section{Discussion}

The dog in this report initially had a lateral scapulohumeral luxation, as documented on the radiographs obtained on the day the dog sustained the injury, which is typical of dogs that sustain traumatic scapulohumeral luxations (Vaughn, 1967; DeAngelis and Schwartz, 1970; Vasseur et al., 1982, 1983; Vasseur and Gregory, 1985; Brinker et al., 1997). The luxation was reduced in a closed fashion, but maintaining reduction proved problematic. The limb was initially placed in a Velpeau sling which positioned the scapulohumeral joint in flexion and abduction, which likely predisposed the humerus to reluxate. After several recurrences, the limb was placed in a Spica splint, which is the mode of coaptation typically advocated to apply following closed reduction of traumatic lateral scapulohumeral luxations (DeCamp et al., 2016). Unfortunately, the humerus continued to luxate and the dog developed a pattern of chronic intermittent luxations which often occurred several times a day.

The dog was ambulating normally when presented to the UF SAH; however, the owner reported that the dog's shoulder consistently luxated several times each day. The owner became adept at manually reducing the luxation and at other times the luxation spontaneously reduced. Luxation of the left scapulohumeral joint was readily elicited when the shoulder was flexed and proximal pressure was applied to the humerus. When the dog presented to the UF SAH, the humeral head clearly luxated cranially, rather than laterally, to the scapula, but we were unable to ascertain why the direction of the luxation had changed. Although clinical and cadaveric studies have defined specific pathologies associated with traumatic lateral scapulohumeral luxations (Vasseur et al., 1982), the pathology necessary to produce cranial luxation has not been reported. Joint capsule laxity was the only pathology evident at surgery. Arthroscopic evaluation of the joint during surgery could have potentially elucidated further pathology. Cranial scapulohumeral luxations are rare in dogs and are always the result of trauma (Vaughn, 1967; Campbell, 1968; DeAngelis and Schwartz, 1970; Vasseur et al., 1982, 1983; Vasseur and Gregory, 1985; Brinker et al., 1997).

The procedure utilized in the dog in the current report was first described by DeAngelis and Schwartz (1970) and has been cited in a number of small animal surgical textbooks (Vaughn, 1967; Campbell, 1968; DeAngelis and Schwartz, 1970; DeAngelis, 1975; Vasseur et al., 1982; Hohn et al., 1983; Vasseur et al., 1983; Newton and Nunamaker, 1985; Vasseur and Gregory, 1985; Brinker et al., 1997). Initially during surgery, the humeral head repeatedly luxated cranially, particularly when the scapulohumeral joint was flexed. Once the bicipital tendon was transposed into the trough which had been burred in the incised surface of the greater tubercle, the joint became stable. Following closure of the joint capsule, a plate was contoured and placed over the joint capsule subjacent to the cranial articular margin of the proximal humerus, bridging the transposed tendon and providing supplemental fixation to maintain the tendon's position (Hasiuk et al., 2017). The functionality of this plate is uncertain and the presence of the plate necessitated distal transposition of the greater tubercle when reattached. Distal transposition of the greater tubercle likely increased tension in the supraspinatus muscle which may have augmented the cranial stability of the joint.

The dog in the current report had a profound lameness, loss of muscle mass, and a reduced range of motion of the scapulohumeral joint 1 month following the surgery. Multimodal rehabilitative therapy was implemented to promote tissue healing, maximize range of motion, and facilitate gait retraining (Shmalberg and Memon, 2015). Rehabilitation therapy can optimize tissue repair, increase strength and flexibility, and improve limb function following orthopedic surgery (Marsolais et al., 2002). The modalities utilized in the dog in the current report include: therapeutic ultrasound, photobiomodulation, underwater treadmill, and therapeutic exercise. Therapeutic ultrasound is commonly implemented in rehabilitative protocols treating joint contracture, using acoustic energy to heat periarticular tissues and facilitate mobilization (Levine and Watson, 2014). Ultrasound was applied to the left scapulohumeral joint, followed by manual stretching to maximize range of motion. Photobiomodulation therapy was also employed for its analgesic and antiinflammatory properties, and to increase joint mobility and function (Brosseau et al., 2000) benefits of the buoyancy and resistance of water to minimize pain and maximize functional shoulder movement (Speer et al., 1993). The owner was directed to perform targeted athome exercises prescribed to promote weight-bearing and maintenance of range of motion. Although research to determine optimal post-operative rehabilitation protocols in dogs is sparse, particularly with respect to surgical procedures involving scapulohumeral joint, limb function improved substantially in the dog in the current report once aggressive rehabilitative therapy was initiated.

We were particular interested in obtaining an objective assessment of the long-term functional outcome (Cook et al., 2010) of the dog in the current report as assessment of the dog in the original report in which this procedure was used was limited to a 1-month evaluation, at which time the dog was bearing some weight on the limb, and communications with the owner 14 weeks after surgery, at which time the owner reported that lameness had resolved (DeAngelis and Schwartz, 1970). The procedure is involved with substantial associated iatrogenic trauma and entraps the bicipital tendon in an osseous tunnel; long-term outcomes following this 
procedure have not be previously reported. Although the dog in the current report had decreased extension of the left scapulohumeral joint, the dog had resumed all normal activities without apparent lameness when evaluated 14 months after surgery. The shoulder was stable and not painful on manipulation. Force plate parameters were slightly lower in the left thoracic limb $(95 \%$ of the peak vertical force of the right limb and $94 \%$ of the peak vertical impulse of the right limb) compared to the right thoracic limb, with slight measurable muscle asymmetry which we partially ascribed to the dog's chronicity of lameness prior to surgery and prolonged coaptation in the initial postoperative convalescent period. There was minimal, progressive scapulohumeral joint degeneration and no appreciable clinical abnormalities attributable to the broken screws. Although transposition of the bicipital tendon resolved this dog's cranial luxation and yielded excellent long-term function, the placement of prostheses or other stabilization techniques (Prostredny et al., 1993; Fitch et al., 2001; Ringwood et al., 2001; O'Donnell et al., 2017) which would likely have less associated iatrogenic trauma should be evaluated to manage dogs with a cranial scapulohumeral luxation in an effort to mitigate postoperative morbidity.

\section{Acknowledgments}

Discretionary funds from the University of Florida Collaborative Orthopedic and Biomechanics Laboratory were used to obtain the dog's long-term follow-up information.

\section{Conflict of interest}

The authors declare that there is no conflict of interest. Author contributions

Dr. Barber and Dr. Lewis were responsible for the primary case management. Dr. Porter was the consulting radiologist of the case and Elam directed the dog's rehabilitation. All four of the authors contributed to writing and editing the manuscript.

\section{References}

Brinker, W.O., Piermattei, D.L. and Flo, G.L. 1997. In Handbook of small animal orthopedics and fracture repair, 3rd ed. Eds., Piermattei, D.L., Flo, G.L. Philadelphia, PA: WB Saunders, p: 237.

Brosseau, L., Welch, V., Wells, G., Tugwell, P., Bie, R., Gam, A., Harman, K., Shea, B. and Morin, M. 2000. Low level laser therapy for osteoarthritis and rheumatoid arthritis: a meta-analysis. J. Rheumatol. 27, 1961-1969.

Campbell, J.R. 1968. Shoulder lameness in the dog. J. Small Anim. Pract. 9, 189-198.

Cook, J.L., Evans, R., Conzemius, M.G., Lascelles, B.D.X., McIlwraith, C.W., Pozzi, A., Clegg, P., Innes, J., Schulz, K., Houlton, J., Fortier, L., Cross, A.R., Hayashi, K., Kapatkin, A., Brown, D.C. and Stewart, A. 2010. Proposed definitions and criteria for reporting time frame, outcome, and complications for clinical orthopedic studies in veterinary medicine. Vet. Surg. 39, 905-908.

DeAngelis, M.P. 1975. Luxations of the shoulder joint. In Current techniques in small animal surgery. Ed., Bojarb, M.J. Philadelphia, PA: Lea and Febiger, pp: 499-504.

DeAngelis, M.P. and Schwartz, A. 1970. Surgical correction of cranial dislocation of the scapulohumeral joint in a dog. J. Am. Vet. Med. Assoc. 156, 435-438.

DeCamp, C.E., Johnston, S.P., Dejarden, L.M., Johnston, S.A. and Schaefer, S.L. 2016. Brinker, piermattei and flo's handbook of small animal orthopedics and fracture repair, 5th ed. St. Louis, MO: Elsevier, Inc.

Fitch, R.B., Breshears, L., Staatz, A. and Kudnig, S. 2001. Clinical evaluation of prosthetic medial glenohumeral ligament repair in the dog (ten cases). Vet. Comp. Orthop. Traumatol. 14, 222-228.

Hasiuk, M.M., Drygas, K.A. and Lewis, D.D. 2017. Long-term outcomes following plate stabilization to address spontaneous luxation of the long digital extensor tendon of origin in 2 dogs. Can. Vet. J. 58, 1176-1180.

Hohn, R.B., Craig, E. and Anderson, W.D. 1983. Luxation of the shoulder joint. In Current techniques in small animal surgery, 2nd ed. Ed., Bojarb, M.J. Philadelphia, PA: Lea and Febiger, pp: 726-733.

Levine, D. and Watson, T. 2014. Therapeutic ultrasound. In: Canine rehabilitation and physical therapy, 2nd ed. Eds., Millis, D.L. and Levine, D. St. Louis, MO: Elsevier Inc, pp: 328-341.

Marsolais, G.S., Dvorak, G. and Conzemius, M.G. 2002. Effects of postoperative rehabilitation on limb function after cranial cruciate ligament repair in dogs. J. Am. Vet. Med. Assoc. 220, 1325-1330.

Newton, C.D. and Nunamaker, D.M. 1985. Textbook of small animal orthopaedics. Philadelphia, PA: JB Lippincott Company, pp: 343-355.

O'Donnell, E.M., Canapp, S.O. Jr., Cook, J.L. and Pike, F. 2017. Treatment of medial shoulder joint instability in dogs by extracapsular stabilization with a prosthetic ligament: 39 cases (2008-2013). J. Am. Vet. Med. Assoc. 251, 1042-1052.

Prostredny, J.M., Toombs, J.P. and Jakovljevic, S. 1993. Use of polybutester suture to repair medial scapulohumeral luxation in the dog: three cases. J. Am. Anim. Hosp. Assoc. 29, 180-183.

Ringwood, P.B., Kerwin, S.C., Hosgood, G. and Williams, J. 2001. Medial glenohumeral ligament reconstruction for ex vivo medial glenohumeral luxation in the dog. Vet. Comp. Orthop. Traumatol. 14, 196-200.

Shmalberg, J. and Memon, M.A. 2015. A retrospective analysis of 5,195 patient treatment sessions in an integrative veterinary medicine service: patient 
characteristics, presenting complaints, and therapeutic interventions. Vet. Med. Int. 983261, 1-11; doi: 10.1155/2015/983621.

Speer, K.P., Cavanaugh, J.T., Warren, Day, L. and Wickiewicz, T.L. 1993. A role for hydrotherapy in shoulder rehabilitation. Am. J. Sports Med. 21, 850-853.

Vasseur, P.B. and Gregory, C.R. 1985. Luxation of the scapulohumeral joint. In The textbook of small animal surgery. Ed., Slatter, D.H. Philadelphia, PA, WB Saunders, pp: 2056-2061.
Vasseur, P.B., Moore, D. and Brown, S.A. 1982. Stability of the canine shoulder joint: an in vitro analysis. Am. J. Vet. Res. 43, 352-355.

Vasseur, P.B., Pool, R.R. and Klein, K. 1983. Effects of tendon transfer on the canine scapulohumeral joint: an experimental study. Am. J. Vet. Res. 44, 811-815.

Vaughn, L.C. 1967. Dislocation of the shoulder joint in the dog and cat. J. Small Anim. Pract. 8, 45-48. 